\title{
Heme is involved in the systemic inflammatory response following radiofrequency ablation of hepatic hemangiomas
}

\section{Xu Yang}

Beijing Chaoyang Hospital

Jun Liu

Beijing Chaoyang Hospital

Meng-meng Yang

Beijing Chaoyang Hospital

\section{Shao-hong Wang}

Beijing Chaoyang Hospital

Li Xu

Beijing Chaoyang Hospital

Shan Ke

Beijing Chaoyang Hospital

Xue-mei Ding

Beijing Chaoyang Hospital

\section{Wen-bing Sun}

Beijing Chaoyang Hospital

JUN GAO ( $\nabla$ gaojun8430@163.com )

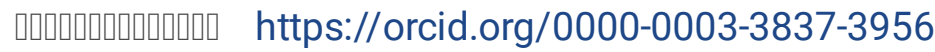

\section{Research article}

Keywords: Heme, Systemic inflammatory response syndrome, Radiofrequency ablation, Hepatic hemangioma

Posted Date: September 24th, 2019

DOl: https://doi.org/10.21203/rs.2.14750/v1

License: (c) (i) This work is licensed under a Creative Commons Attribution 4.0 International License. Read Full License

Version of Record: A version of this preprint was published at European Journal of Gastroenterology \& Hepatology on December 16th, 2019. See the published version at https://doi.org/10.1097/MEG.0000000000001636. 


\section{Abstract}

Background Radiofrequency ablation (RFA) is an effective and minimally invasive treatment for managing hepatic hemangiomas. Systemic inflammatory response syndrome (SIRS) often occurs with hemoglobinuria, and its underlying pathophysiological mechanism is unknown. Heme can trigger inflammation by inducing the generation of reactive oxygen species (ROS) and the production of inflammatory mediators. We therefore investigated whether circulating heme is involved in SIRS following RFA of hepatic hemangiomas.

Methods We enrolled 65 patients with hepatic hemangioma who underwent RFA. Serum concentrations of free heme, ROS, and tumor necrosis factor a (TNF-a) were measured after RFA. Univariate analysis and a multivariate binary logistic regression model were used to evaluate the contribution of 17 risk factors for SIRS after RFA.

Results Fifty-nine (59/65, 90.8\%) patients developed hemoglobinuria, among which 25 (38\%) experienced SIRS shortly after RFA. In the SIRS group, the serum concentrations of heme, ROS, and TNF-a were immediately elevated after RFA compared with baseline and slowly regained their normal levels 3 days after RFA. Moreover, the concentrations of circulating heme significantly correlated with those of ROS ( $r=0.805, P<0.001)$ and TNF$a(r=0.797, P<0.001)$. Univariate analysis showed that the volume of a hemangioma $(O R=1.293, P=0.031)$, time of ablation $(O R=1.194, P=0.029)$ as well as the concentrations of heme $(O R=1.430, P=0.017)$, ROS $(\mathrm{OR}=1.251, \mathrm{P}=0.031)$, and TNF- $\mathrm{a}(\mathrm{OR}=1.309, \mathrm{P}=0.032)$ were significantly associated with SIRS.

Conclusions Circulating heme was associated with the induction of ROS and the production of TNF- $a$, which may contribute to the induction of SIRS following RFA of hepatic hemangiomas.

\section{Background}

Hepatic hemangiomas are the most common benign tumors of the liver, with an incidence ranging from $0.4 \%$ to $20 \%$ among the general population $[1,2]$. Fortunately, most lesions are small, asymptomatic, and do not require surgical intervention [2]. However, giant hemangiomas (diameters $\geq 5 \mathrm{~cm}$ ) pose a higher risk of rupture, which presents with uncontrollable abdominal pain. The size of such lesions may increase during follow-up, potentially requiring radical intervention $[3,4]$.

Radiofrequency ablation (RFA) is increasingly used for managing hepatic hemangiomas because of its unique advantages compared with other therapies. The advantages include minimal invasiveness, low cost, low incidence of complications, short duration of hospitalization, and increased patient compliance [5-7]. However, the nearly unavoidable hemolysis after RFA, attributable to the generous blood supply of hepatic hemangiomas, is a major disadvantage. Moreover, the incidence of systemic inflammatory response syndrome (SIRS) is high among patients who experience hemoglobinuria after RFA and may be accompanied by acute respiratory distress syndrome and severe myocardial dysfunction $[7,8]$. Unfortunately, the underlying pathophysiological mechanism is unknown.

Intravascular hemolysis, or the destruction of red blood cells (RBCs) in the circulation, can occur in numerous diseases, including the acquired hemolytic anemias, sickle cell disease, and thalassemia, as well as during transfusion reactions, pre-eclampsia, and infections. During hemolysis, heme derived from hemoglobin $(\mathrm{Hb})$ accumulates because of the inability of detoxification systems to sufficiently scavenge it [9]. Evidence indicates 
that $\mathrm{Hb}$-derived heme may serve as an endogenous danger signal, triggering inflammation whenever red blood cells (RBCs) are destroyed, and $\mathrm{Hb}$ is released into extracellular compartments $[10,11]$.

Free heme mediates diverse pathological effects, including increased production of reactive oxygen species (ROS) and inflammatory mediators such as TNF- $囚$ as well as upregulation of the production of endothelial cell adhesion molecules [12-16]. These factors can lead to inflammation in sterile and infectious conditions, contributing to the pathogenesis of hemolytic diseases, subarachnoid hemorrhage, malaria, and sepsis [12, 13]. However, we are unaware of studies that address whether heme contributes to the incidence of SIRS after patients undergo RFA to treat hepatic hemangioma. The goal of the present study is to answer this question.

\section{Materials And Methods}

\section{Patients and blood sample collection}

From January 2016 to December 2018, 65 patients with hepatic hemangiomas underwent RFA at our institution. The inclusion criteria for RFA are described in our previous study [17]. These patients did not experience significant heart, lung, liver, kidney abnormities, or other serious concomitant diseases before undergoing RFA.

RFA was performed using internally cooled cluster electrodes, Cool-tip ACTC 2025 (for laparoscopic procedures) or ACTC 1525 (for CT-guided percutaneous procedures) electrodes, and an RF generator (Covidien Healthcare, Dublin, Ireland).

Blood cell count, C-reactive protein (CRP), urine analysis, and routine biochemical tests were used to evaluate liver and renal function before RFA and $1 \mathrm{~h}$ and $1-3$ days after RFA. Chemical analysis of $\mathrm{Hb}$ and flow cytometry were used to detect RBCs. Hemoglobinuria was diagnosed if the $\mathrm{Hb}$ and RBC tests were positive and negative, respectively [18]. Wine-colored hemoglobinuria can be visually observed, and deep-yellow hemoglobinuria can only be detected using routine urine analysis. Blood samples were collected in heparinized tubes before RFA and $1 \mathrm{~h}$ and $1-3$ days after RFA. After sampling, the serum was prepared using centrifugation, divided into aliquots, and stored at $-70{ }^{\circ} \mathrm{C}$ for testing serum concentrations of heme, ROS, and TNF-a.

All patients granted written informed consent before treatment. The Research and Ethics Committee of Beijing Chao-yang Hospital, Capital Medical University approved this study, which was conducted in accordance with the standards of the Declaration of Helsinki.

\section{Definition of SIRS}

SIRS was diagnosed according to findings of least two of the variables as follows: body temperature $>38{ }^{\circ} \mathrm{C}$ or $<36{ }^{\circ} \mathrm{C}$, heart rate $>90 \mathrm{bpm}$, respiratory rate $>20$ breaths $/ \mathrm{min}$ or $\mathrm{PaCO}^{2}<32 \mathrm{mmHg}$, and WBC count $>12 \times 10^{9} / \mathrm{L}$ or $<4 \times 10^{9} /$ L [19].

\section{Ablated volume of a hemangioma}


The ablated volume of a hemangioma, which was considered equivalent to the volume of the hemangioma before RFA, was determined according to the results of contrast-enhanced computed tomography (CT) or magnetic resonance imaging (MRI) before RFA to correlate the ablated volume with SIRS. Lesion volumes were calculated as follows: volume $=X \times Y \times Z \times \pi / 6$, where $X, Y$, and $Z$ are the maximum diameters (supine vertical, sagittal, and coronal planes) of the tumor measured using CT or MRI [20].

\section{Histology of Excised Hemangiomas}

Hemangioma tissues were laparoscopically resected after RFA [21]. The tissues in the region of and "adjacent ablated hepatic hemangioma," located $<1.0 \mathrm{~cm}$ from the ablation tissues were collected. Hepatic hemangiomas and adjacent ablated hepatic hemangiomas were fixed using $4 \%$ buffered paraformaldehyde, dehydrated, and embedded in paraffin. Sections (5- $\mu \mathrm{m}$ thick) were deparaffinized, rehydrated, and rinsed in distilled water. The tissues were deparaffinized and stained with hematoxylin-eosin (HE).

\section{Measurements of heme, ROS and TNF-a]}

We passed serum samples through a Microcon YM-3 column (Millipore, Solarbio, Beijing, China) (60 min at 14 ${ }^{\circ} \mathrm{C}, 21000 \mathrm{~g}$ ) to remove proteins $>3 \mathrm{kDa}$. We quantified free heme in these protein-depleted sera using a chromogenic assay in accordance with the manufacturer's instructions (QuantiChrom Heme Assay Kit, Bioassay Systems, California, USA).

The amounts of free radicals in serum samples were measured using the OxiSelect In Vitro ROS/RNS Assay Kit (Cell Biolabs, San Diego, USA) that uses stabilized dichloro-dihydro-fluorescein (DCFH) instead of DCFHdiacetate. Stabilized, highly reactive DCFH is oxidized by ROS in the presence of a proprietary catalyst provided by the manufacturer to help accelerate the reaction. Serum samples were diluted in phosphate-buffered saline (1: 100), and $50-\mu \mathrm{l}$ of the diluted samples were assayed in accordance with the manufacturer's protocol. The ratio of the fluorescence intensities of oxidized DCFH to DCF is proportional to the concentration of ROS. Fluorescence was measured at $492 \mathrm{~nm}$ excitation/535 nm emissions using a Wallac Victor fluorescence plate reader (Perkin Elmer, Massachusetts, USA). Serum TNF-a囚 concentrations were measured using an enzymelinked immunosorbent assay (Elabscience, Wuhan, China) in accordance with the manufacturers' protocol.

\section{Statistical analysis}

The values of continuous variables are presented as the mean \pm standard deviation (SD) or median values with interquartile ranges, and those of categorical variables are presented as percentages. Categorical data were compared using the Chi-square test or Fisher's exact test, and continuous data were compared using the Student $t$ test or the Wilcoxon rank-sum test. Pearson's correlation coefficient was determined to assess the significance of correlations between variables. Univariate and multivariate logistic regression analyses were conducted with the diagnosis of SIRS as a dependent variable. Only parameters that were significantly associated with SIRS identified by univariate logistic analysis were included in the multivariate logistic regression model, and $P<0.05$ indicates a significant difference. Statistical analysis was performed using 
SPSS version 17.0 for Windows (SPSS, Chicago, IL, USA). All descriptive graphs were generated using GraphPad Prism version 5.00 for Windows (GraphPad Software, San Diego, USA).

\section{Results}

\section{Patients' characteristics}

RFA was successful for all patients. Of 65 patients, 59 (90.8\%) developed hemoglobinuria shortly after RFA. Subsequently, the color of urine, or that determined using routine urine analysis, recovered gradually after reaching baseline values and hydration treatment in 1-2 days. Major complications caused by acute kidney injury occurred. Of 59 patients with hemoglobinuria, $25(42 \%)$ patients experienced SIRS. Fluid replacement, dieresis, and glucocorticoids were administered, and 24 (96.0\%) patients recovered within 3-4 days. One patient developed severe myocardial dysfunction. Chest X-ray showed the butterfly sign of alveolar edema with cardiomegaly. Therapy was immediately initiated, including high-flow oxygen inhalation delivered through a face mask, intravenous (IV) diuretics, IV methylprednisolone, and short-term antibiotic treatment, after which clinical symptoms gradually disappeared, and heart and lung function slowly improved. This patient was discharged at 9 days after RFA. Long-term sequelae were not observed in patients during the 6-month follow-up.

All patients were classified according to the diagnostic criteria stated above into the SIRS group or Non-SIRS group. The patients' clinical parameters are summarized in Table 1.

\section{Hemolysis in adjacent ablated hepatic hemangiomas}

In hepatic hemangioma tissue, blood vessels were characterized by spongy hyperplasia, endothelial cells were arranged along the vascular wall, and the RBCs in the lumen were normal (HE, 200x) (Figure 1A). The "adjacent ablated hepatic hemangioma" tissue did not exhibit coagulative necrosis, the endothelial cells were disordered along the wall of the blood vessels, and partial burning and deformation of blood cells in the were accompanied by their destruction in the lumen (HE, 200x) (Figure 1B).

\section{Changes of heme, ROS and TNF- $a$ in patients with hemangioma}

In the SIRS group, increased concentrations of serum heme $(7.72 \pm 0.61 \mu \mathrm{M})$ were detected $1 \mathrm{~h}$ after ablation compared with preoperative concentrations $(0.49 \pm 0.31 \mu \mathrm{M})$ and slowly decreased from the day after RFA. The peak value occurred $1 \mathrm{~h}$ after RFA. In the non-SIRS group, heme concentrations after RFA were slightly higher compared with those before RFA, but the difference was not significant (Figure. 2A).

Compared with ROS concentrations before RFA $(19.54 \pm 2.96 \mu \mathrm{M})$, those $1 \mathrm{~h}$ after ablation were significantly increased in the SIRS group $(71.07 \pm 5.33 \mu \mathrm{M}, P<0.001)$. ROS concentrations decreased to pre-RFA concentrations 3 days later $(20.92 \pm 2.33 \mu \mathrm{M})$, although the difference was not significant (Figure 2B). ROS concentrations peaked $1 \mathrm{~h}$ after RFA in the non-SIRS group, and ROS concentrations were slightly higher (20.92 $\pm 3.42 \mu \mathrm{M}$ ) after RFA, although the difference was not significant (Figure 2B). 
In the SIRS group, serum concentrations of TNF-a were significantly increased $1 \mathrm{~h}$ after RFA, and those of TNF- $\mathrm{a}$ increased 10-fold $(20.07 \pm 2.12 \mathrm{pg} / \mathrm{mlvs} 1.98 \pm 0.64 \mathrm{pg} / \mathrm{ml})$. Three days after RFA, TNF-a concentrations decreased $(2.29 \pm 1.00 \mathrm{pg} / \mathrm{ml})$, but remained above pre-RFA concentrations, although the difference was not significant (Figure 2C). TNF-a concentrations peaked $1 \mathrm{~h}$ after RFA. In the non-SIRS group, the changes in TNF-a concentrations were not significantly different after RFA (Figure 2C).

To investigate whether there was an association between circulating heme concentrations and heme-related factors, Pearson's correlation coefficients were determined. Circulating heme concentrations were significantly associated with those of ROS $(r=0.805, P<0.001)$ (Figure 3A) and TNF-a $(r=0.797, P<0.001)$ (Figure. 3B).

\section{Risk factors of SIRS}

Univariate analysis identified the volume of a hemangioma $(P=0.022)$, time of ablation $(P=0.015)$, AST $(P=$ $0.020)$, total bilirubin $(P=0.014)$, CRP $(P=0.041)$, heme $(P=0.007)$, ROS $(P=0.038)$, and TNF-a $\mathbb{X}(P=0.014)$ as significant risk factors for the occurrence of SIRS. Multivariate analysis identified the volume of a hemangioma $(P=0.031$, odds ratio $[\mathrm{OR}]=1.293,95 \%$ confidence interval $[\mathrm{Cl}]=0.943-1.746)$, time of ablation $(P=0.029, \mathrm{OR}$ $=1.194,95 \% \mathrm{Cl}=0.838-1.529)$, heme $(P=0.017, \mathrm{OR}=1.430,95 \% \mathrm{Cl}=0.953-2.145), \mathrm{ROS}(P=0.031, \mathrm{OR}=$ $1.251,95 \% \mathrm{Cl}=0.579-3.423), \mathrm{TNF}-\mathrm{a} \rrbracket(P=0.032, \mathrm{OR}=1.309,95 \% \mathrm{Cl}=0.983-3.536)$ as significant independent risk factors associated with SIRS after RFA (Table 2 ).

\section{Discussion}

Traditionally, surgical resection and surgical enucleation are the most frequently used treatments of choice for hepatic hemangiomas. However, it is important to consider that many of the trade-offs encountered during the treatment of cancer are not applicable to hepatic hemangiomas because of their benign natural history. Thus, a highly effective but morbid treatment would represent a poor choice for most patients. RFA is safe, well tolerated, and effective for treating most patients with hepatic hemangiomas [4-6]. However, the main disadvantage of RFA is the nearly unavoidable hemolysis attributable to the generous blood supply of a hepatic hemangioma [17]. For example, depending on the severity of hemolysis, hemoglobinuria, hemolytic jaundice, anemia, or renal damage can occur [7, 8]. Further, adverse events associated with SIRS often occur in patients with hemoglobinuria after they undergo RFA to treat hepatic hemangioma, particularly for hemangiomas $\geq 10$ $\mathrm{cm}$. Whether hemolysis and SIRS are pathologically associated is unknown. Here we discovered an important contribution of heme to RFA-induced SIRS.

Heme is a ubiquitous molecular complex of iron and the tetrapyrrole protoporphyrin IX [12, 13]. When bound to hemoproteins, heme plays an essential role in numerous biological processes in aerobic organisms, such as from reversible binding of oxygen to electron transport molecules of the respiratory chain [12]. After hemolysis, extracellular $\mathrm{Hb}$ is readily oxidized by ferrous $\mathrm{Hb}$ to yield ferric $\mathrm{Hb}$ (methemoglobin), which readily releases free heme. Evidence supports the conclusion that inflammatory mechanisms driven by heme may play a fundamental role in the pathophysiology of hemolytic diseases [16, 22]. For example, heme is directly cytotoxic and can activate specific receptors and signaling pathways to promote the generation of ROS and induce inflammation and programed cell death $[9,16,23]$. Studies using models of infectious and noninfectious diseases show that heme can induce monocytes and macrophages to secrete TNF-a through TLR4-mediated 
signaling, which induces inflammation $[14,15,24]$. These results highlight the great potential importance of studies of the molecular mechanisms of heme-induced inflammation and cell death aimed on identifying new therapeutic targets.

In the present study, pathology verified that RFA of hepatic hemangiomas led to massive destruction of RBCs and intravascular hemolysis. Specifically, 59 patients developed hemoglobinuria, among which 25 experienced SIRS. We detected significant increases in the concentrations of circulating heme, ROS, and TNF-a in the SIRS group $1 \mathrm{~h}$ after ablation, which decreased to pre-RAF concentrations after 3 days. Further, the concentrations of circulating heme were significantly associated with those of ROS and TNF-a. Multivariate analysis identified baseline patients' characteristics $1 \mathrm{~h}$ after undergoing RFA that were associated with the risk of SIRS such as sex, age, concomitant illness, volume of a hemangioma, distribution of the lesion, RFA, time of RFA, hemoglobinuria, and the concentrations of ALT, AST, total bilirubin, BUN, creatinine, CRP, heme, ROS, and TNF-a. Multivariate analysis showed that the volume of a hemangioma, time of RFA, heme, ROS, and TNF-a were independent risk factors of SIRS. We thus raise the possibility that the release of heme from damaged RBCs caused by RFA induces SIRS, which represents a novel mechanism of RFA-related SIRS.

However, there are limitations to the present study. First, the sample size was relatively small, which decreased statistical power. Second, cell culture and animals model of hepatic hemangioma are required to mimic clinical RFA. Third, our results are from a single center, which may have introduced selection bias that may explain our results.

\section{Conclusions}

We found that damage to RBCs caused by RFA released extracellular heme to the peripheral circulation, which induced the production of ROS and inflammatory cytokines that may contribute to SIRS after RFA of hepatic hemangiomas. Recognition of the inflammatory burden of hemolytic processes during this treatment will likely serve as a foundation for developing new approaches for use in combination with established therapies aimed at treating hemolytic diseases, with the goal of diminishing or neutralizing the effects of the release of $\mathrm{Hb}$ and heme by damaged RBCs.

\section{Declarations}

\section{Abbreviations}

SIRS: ystemic inflammatory response syndrome; RFA: Radiofrequency ablation; ROS: reactive oxygen species; TNF-a: tumor necrosis factor a

\section{Ethics approval and consent to participate}

All procedures performed in studies involving human participants were in accordance with the ethical standards of the institutional research committee and with the 1964 Helsinki declaration and its later amendments or comparable ethical standards. The Institutional Review Board of Beijing Chao-yang Hospital approved this 
research. All participants agreed to the study conditions and provided written informed consents before the enrollment in this study.

\section{Consent for publication}

Not applicable

\section{Availability of data and materials}

The data used and analyzed during the current study are included in this published article, and are also available from the corresponding author on reasonable request. Technical appendix, statistical code, and dataset are also available from the corresponding author on reasonable request.

\section{Competing interests}

The authors declare that they have no competing interests.

\section{Funding}

This study was supported by the program for high-level technical talents in Beijing health system冈2015-03025区.

\section{Authors' contributions}

JG designed the study; XY and JL did literature search and wrote the paper; MMY performed enzyme linked immunosorbent assay; LX, SK, XMD and WBS carried out RFA treatment; SHW carried out the data angalysis; JG revised the manuscript. All authors read and approved the final manuscript.

\section{Acknowledgements}

\section{We acknowledge Shu-Ying Dong for help in collecting serum samples of patients.}

\section{References}

1.Yamashita S, Okita K, Harada K, et al. Giant cavernous hepatic hemangioma shrunk by use of sorafenib. Clin J Gastroenterol, 2013, 6(1): 55-62. DOI: 10.1007/s12328-012-0343-0.

2.Hasan HY, Hinshaw JL, Borman EJ, et al. Assessing normal growth of hepatic hemangiomas during long-term follow-up. JAMA Surg, 2014, 149(12): 1266-71. DOI: 10.1001/jamasurg.2014.477. 
3.Miura JT, Amini A, Schmocker R, et al. Surgical management of hepatic hemangiomas: a multi-institutional experience. HPB (Oxford), 2014, 16(10): 924-8. DOI: 10.1111/hpb.12291.

4.Zhang W, Huang ZY, Ke CS, et al. Surgical Treatment of Giant Liver Hemangioma Larger Than $10 \mathrm{~cm}$ : A Single Center's Experience With 86 Patients. Medicine (Baltimore), 2015, 94(34): e1420. DOI:

10.1097/md.0000000000001420.

5.Park SY, Tak WY, Jung MK, et al. Symptomatic-enlarging hepatic hemangiomas are effectively treated by percutaneous ultrasonography-guided radiofrequency ablation. J Hepatol, 2011, 54(3): 559-65. DOI:

10.1016/j.jhep.2010.07.024.

6.Gao J, Ke S, Ding XM, et al. Radiofrequency ablation for large hepatic hemangiomas: initial experience and lessons. Surgery, 2013, 153(1): 78-85. DOI: 10.1016/j.surg.2012.06.004.

7.Gao J, Ji JS, Ding XM, et al. Laparoscopic Radiofrequency Ablation for Large Subcapsular Hepatic Hemangiomas: Technical and Clinical Outcomes. PLoS One, 2016, 11(2): e0149755. DOI:

10.1371/journal.pone.0149755.

8.Gao J, Xu L, Yang MM, et al. A Severe Complication of Myocardial Dysfunction Post Radiofrequency Ablation Treatment of Huge Hepatic Hemangioma: A Case Report and Literature Review. Open Med (Wars), 2019, 14: 398-402. DOI: 10.1515/med-2019-0041.

9.Mendonca R, Silveira AAConran N Red cell DAMPs and inflammation. Inflamm Res, 2016, 65(9): 665-78. DOI: 10.1007/s00011-016-0955-9.

10.Schaer DJ, Buehler PW, Alayash Al, et al. Hemolysis and free hemoglobin revisited: exploring hemoglobin and hemin scavengers as a novel class of therapeutic proteins. Blood, 2013, 121(8): 1276-84. DOI: 10.1182/blood2012-11-451229.

11.Gouveia Z, Carlos AR, Yuan X, et al. Characterization of plasma labile heme in hemolytic conditions. Febs j, 2017, 284(19): 3278-3301. DOI: 10.1111/febs.14192.

12.Larsen R, Gouveia Z, Soares MP, et al. Heme cytotoxicity and the pathogenesis of immune-mediated inflammatory diseases. Front Pharmacol, 2012, 3: 77. DOI: 10.3389/fphar.2012.00077.

13.Dutra FFBozza MT Heme on innate immunity and inflammation. Front Pharmacol, 2014, 5: 115. DOI: 10.3389/fphar.2014.00115.

14.Belcher JD, Chen C, Nguyen J, et al. Heme triggers TLR4 signaling leading to endothelial cell activation and vaso-occlusion in murine sickle cell disease. Blood, 2014, 123(3): 377-90. DOI: 10.1182/blood-2013-04495887.

15.Erdei J, Toth A, Balogh E, et al. Induction of NLRP3 Inflammasome Activation by Heme in Human Endothelial Cells. 2018, 2018: 4310816. DOI: 10.1155/2018/4310816.

16.Guarda CCD, Santiago RP, Fiuza LM, et al. Heme-mediated cell activation: the inflammatory puzzle of sickle cell anemia. Expert Rev Hematol, 2017, 10(6): 533-541. DOI: 10.1080/17474086.2017.1327809. 
17.Gao J, Fan RF, Yang JY, et al. Radiofrequency ablation for hepatic hemangiomas: A consensus from a Chinese panel of experts. World J Gastroenterol, 2017, 23(39): 7077-7086. DOI: 10.3748/wjg.v23.i39.7077.

18.Veerreddy P Hemoglobinuria misidentified as hematuria: review of discolored urine and paroxysmal nocturnal hemoglobinuria. Clin Med Insights Blood Disord, 2013, 6: 7-17. DOI: 10.4137/cmbd.s11517.

19.Bone RC, Balk RA, Cerra FB, et al. Definitions for sepsis and organ failure and guidelines for the use of innovative therapies in sepsis. The ACCP/SCCM Consensus Conference Committee. American College of Chest Physicians/Society of Critical Care Medicine. Chest, 1992, 101(6): 1644-55. DOI: 10.1378/chest.101.6.1644.

20.Dong S, Kong J, Kong F, et al. Insufficient radiofrequency ablation promotes epithelial-mesenchymal transition of hepatocellular carcinoma cells through Akt and ERK signaling pathways. J Transl Med, 2013, 11: 273. DOI: $10.1186 / 1479-5876-11-273$.

21.Wang S, Gao J, Yang M, et al. Intratumoral coagulation by radiofrequency ablation facilitated the laparoscopic resection of giant hepatic hemangioma: a surgical technique report of two cases. Oncotarget, 2017, 8(31): 52006-52011. DOI: 10.18632/oncotarget.18994.

22.Lee SKDing JL A perspective on the role of extracellular hemoglobin on the innate immune system. DNA Cell Biol, 2013, 32(2): 36-40. DOI: 10.1089/dna.2012.1897.

23.Kato GJ, Steinberg MHGladwin MT Intravascular hemolysis and the pathophysiology of sickle cell disease. J Clin Invest, 2017, 127(3): 750-760. DOI: 10.1172/jci89741.

24.Vinchi F, Costa da Silva M, Ingoglia G, et al. Hemopexin therapy reverts heme-induced proinflammatory phenotypic switching of macrophages in a mouse model of sickle cell disease. Blood, 2016, 127(4): 473-86. DOI: 10.1182/blood-2015-08-663245.

\section{Tables}

Table I. Comparison of clinical parameters between SIRS group and non-SIRS group patients after RFA for hepatic hemangiomas 


\begin{tabular}{|c|c|c|c|}
\hline & $\begin{array}{l}\text { Non-SIRS } \\
(n=40)\end{array}$ & $\begin{array}{c}\text { SIRS } \\
(n=25)\end{array}$ & $P$ value \\
\hline Age (years) & $50.00 \pm 9.86$ & $49.67 \pm 13.75$ & 0.937 \\
\hline Gender & & & 0.630 \\
\hline Male & $15(37.5 \%)$ & $12(48.0 \%)$ & \\
\hline Female & $25(62.5 \%)$ & $13(52.0 \%)$ & \\
\hline \multicolumn{4}{|l|}{ Co-morbidities, N (\%) } \\
\hline Gallbladder stones & $7(17.5 \%)$ & $4(16.0 \%)$ & 0.764 \\
\hline Type 2 diabetes mellitus & $8(20.0 \%)$ & $4(16.0 \%)$ & 0.764 \\
\hline History of open cholecystectomy & $3(7.5 \%)$ & $1(4.0 \%)$ & 0.808 \\
\hline Chronic hepatitis B & $3(7.5 \%)$ & $2(8.0 \%)$ & 0.523 \\
\hline History of previous liver surgery & $2(5.0 \%)$ & $1(4.0 \%)$ & 0.808 \\
\hline Hepatic cysts & $5(12.5 \%)$ & $6(24.0 \%)$ & 0.149 \\
\hline Volume of hemangiomas $\left(\mathrm{cm}^{3}\right)$ & $94.75 \pm 42.99$ & $262.92 \pm 261.91$ & 0.027 \\
\hline Distribution of lesion, N (\%) & & & 0.817 \\
\hline Right lobe & $18(45.0 \%)$ & $11(44.0 \%)$ & \\
\hline Left lobe & $22(55.0 \%)$ & $14(56.0 \%)$ & \\
\hline Approach of RFA, N (\%) & & & 0.506 \\
\hline Laparoscopic approach & $31(77.5 \%)$ & $21(84.0 \%)$ & \\
\hline Percutaneous approach & $9(22.5 \%)$ & $4(16.0 \%)$ & \\
\hline Time of ablation (min) & $31.52 \pm 8.99$ & $45.67 \pm 16.22$ & 0.007 \\
\hline Hemoglobinuria & $34(85.0 \%)$ & $25(100 \%)$ & 0.074 \\
\hline \multicolumn{4}{|l|}{ Laboratory findings-pre } \\
\hline $\operatorname{ALT}(U / L)$ & $21.85 \pm 12.29$ & $21.63 \pm 14.20$ & 0.964 \\
\hline AST (U/L) & $21.97 \pm 8.83$ & $20.90 \pm 9.06$ & 0.736 \\
\hline Total bilirubin $(\mu \mathrm{mol} / \mathrm{L})$ & $12.90 \pm 3.76$ & $11.47 \pm 4.59$ & 0.339 \\
\hline BUN (mmol/L) & $4.96 \pm 1.47$ & $4.76 \pm 0.99$ & 0.660 \\
\hline
\end{tabular}


Creatinine $(\mu \mathrm{mol} / \mathrm{L})$

$\mathrm{CRP}(\mathrm{mg} / \mathrm{L})$

Laboratory findings-post 1 hour

\begin{tabular}{|c|c|c|c|}
\hline $\operatorname{ALT}(\mathrm{U} / \mathrm{L})$ & $70.43 \pm 29.32128 .27 \pm 83.60$ & $89.95 \pm 22.26$ & 0.044 \\
\hline AST (U/L) & $24.42 \pm 7.77$ & $215.78 \pm 95.06$ & 0.010 \\
\hline Total bilirubin $(\mu \mathrm{mol} / \mathrm{L})$ & $4.08 \pm 0.94$ & $37.86 \pm 15.50$ & 0.006 \\
\hline BUN (mmol/L) & $53.90 \pm 16.72$ & $7.32 \pm 1.53$ & 0.041 \\
\hline Creatinine $(\mu \mathrm{mol} / \mathrm{L})$ & $5.25 \pm 0.77$ & $71.74 \pm 15.72$ & 0.039 \\
\hline $\mathrm{CRP}(\mathrm{mg} / \mathrm{L})$ & & $6.82 \pm 2.49$ & 0.019 \\
\hline \multicolumn{4}{|l|}{ Heme-related index-pre } \\
\hline & $0.50 \pm 0.23$ & $0.49 \pm 0.31$ & 0.272 \\
\hline Free heme $(\mu \mathrm{M})$ & $18.56 \pm 3.76$ & $19.54 \pm 2.96$ & 0.823 \\
\hline $\mathrm{ROS}(\mu \mathrm{M})$ & $1.97 \pm 0.64$ & $1.98 \pm 0.64$ & 0.872 \\
\hline 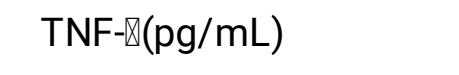 & & & \\
\hline Heme-related index-post 1 & & & \\
\hline Free heme(mg/dl) & $1.10 \pm 0.67$ & $7.72 \pm 0.61$ & 0.017 \\
\hline & $28.09 \pm 5.91$ & $71.07 \pm 5.33$ & 0.001 \\
\hline 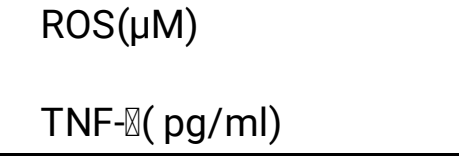 & $3.68 \pm 1.64$ & $20.07 \pm 2.12$ & 0.003 \\
\hline
\end{tabular}

$62.04 \pm 13.52 \quad 65.45 \pm 15.25 \quad 0.508$

$$
\begin{array}{lll}
5.37 \pm 0.81 & 7.02 \pm 7.12 & 0.357
\end{array}
$$

Ictors associated with SIRS from univariate and multivariate logistic regression models 


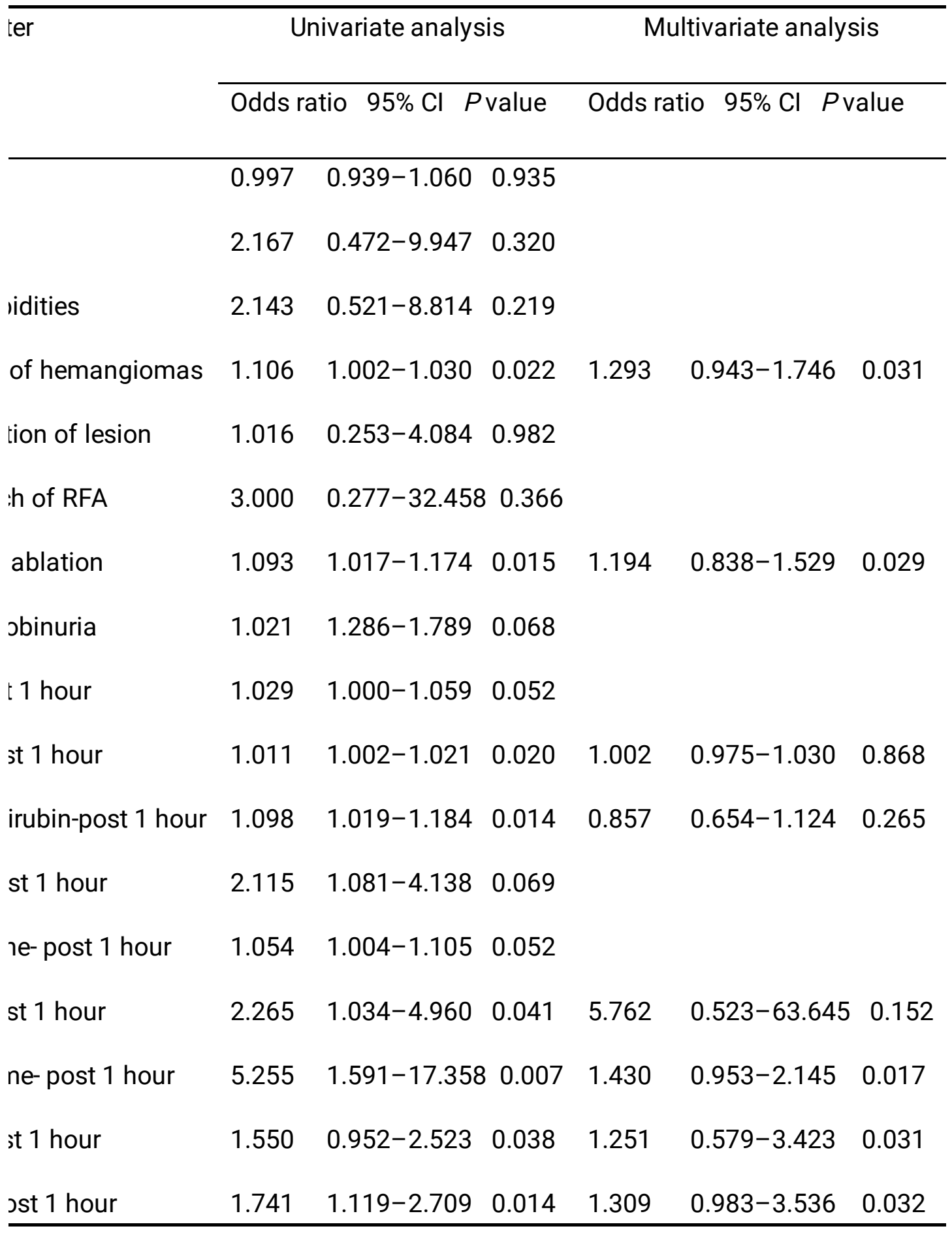

\section{Figures}




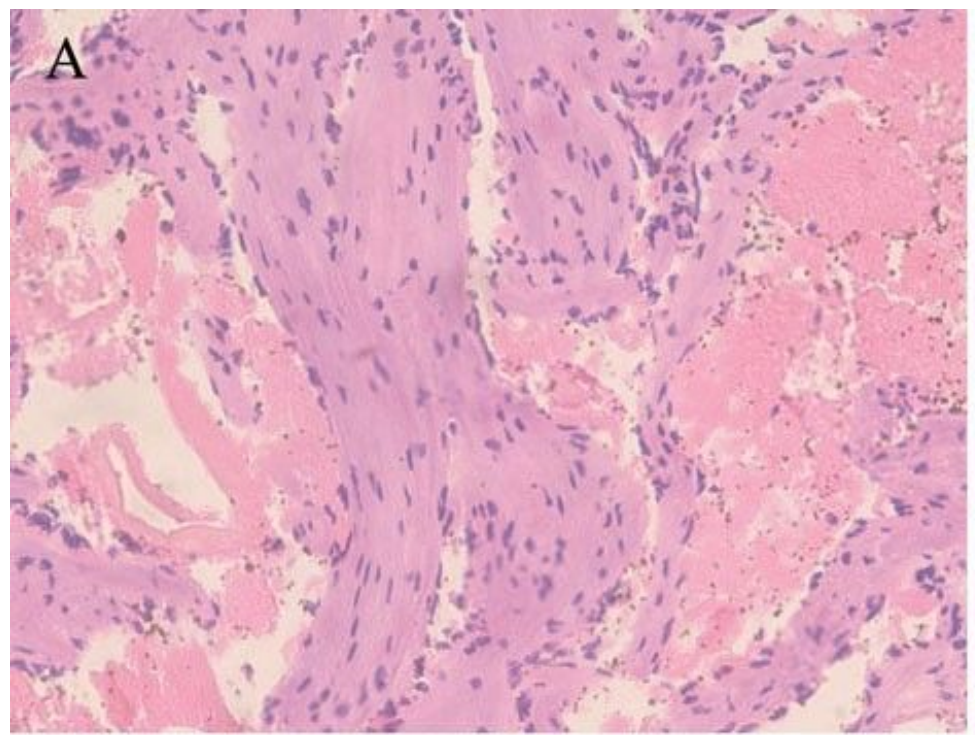

hepatic hemangioma(HE 200x)

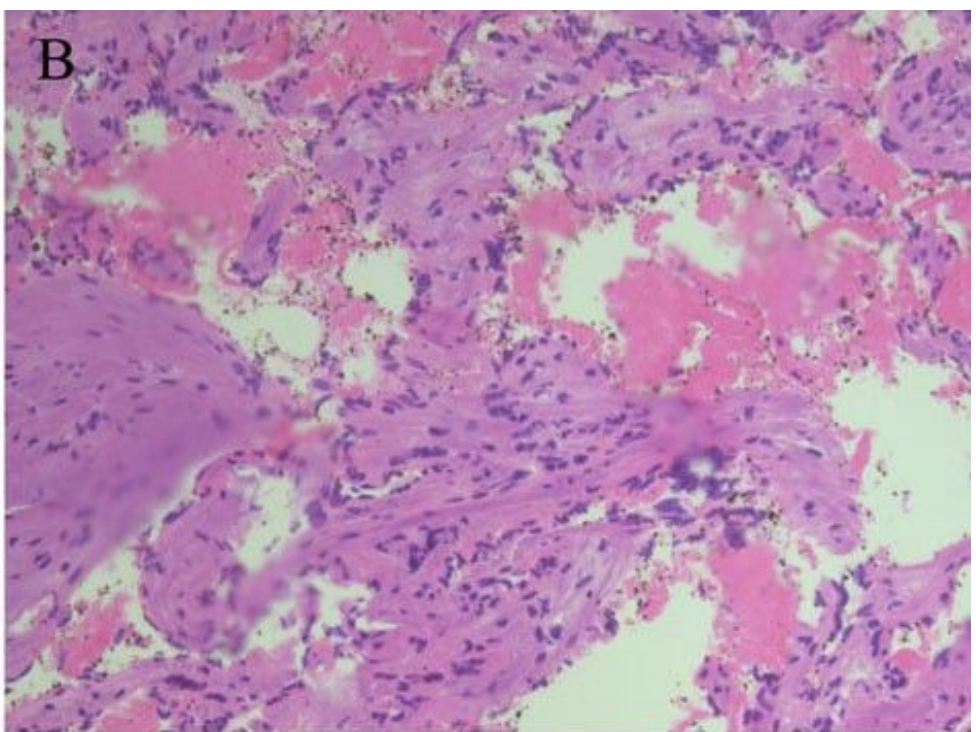

adjacent ablative hepatic hemangioma(HE 200x)

Figure 1. Histology of excised hemangiomas. (A) Hepatic hemangioma tissue showing normal red blood cells in the lumen. (B) Adjacent ablated hepatic hemangioma tissue with red blood cell destruction in the lumen.

Figure 1

A

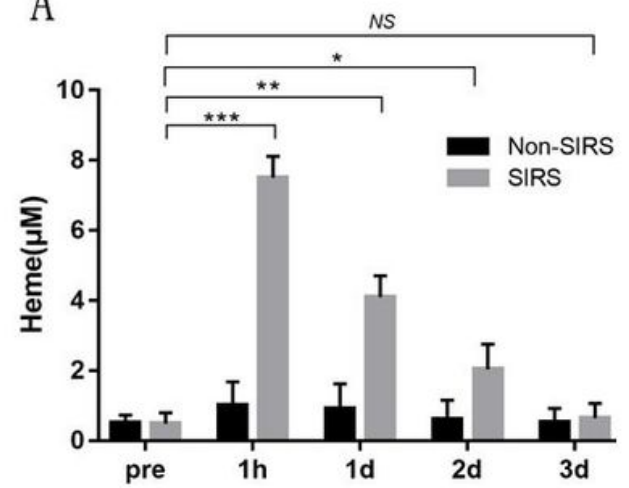

B

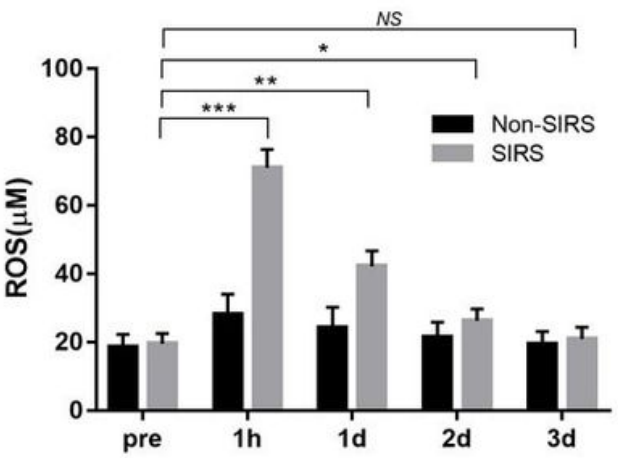

$\mathrm{C}$

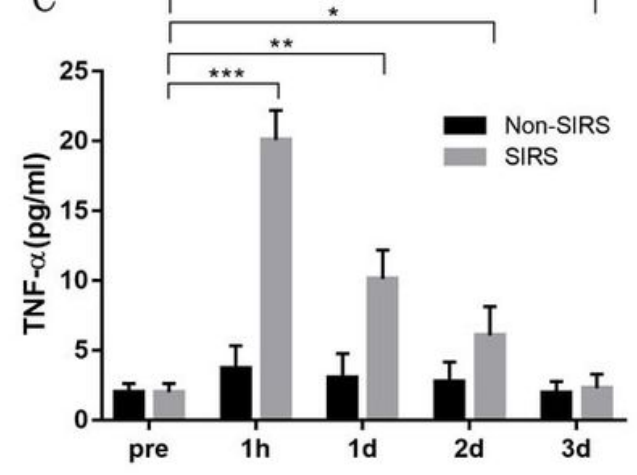

Figure 2. Changes of heme-related parameters in patients with hemangioma. (A) Higher concentrations of serum heme in the SIRS group were detected $1 \mathrm{~h}$ after ablation compared with preoperative levels and were slowly reduced the first day after RFA. The peak concentration occurred $1 \mathrm{~h}$ after RFA. In the non-SIRS group, heme levels after RFA were slightly higher compared with those before RFA, although the difference was not significant. (B) ROS concentrations were significantly increased $1 \mathrm{~h}$ after RFA in the SIRS group and then decreased to those before RFA after 3 days. The peak concentration of ROS was observed $1 \mathrm{~h}$ after RFA. In the non-SIRS group, ROS concentrations were slightly higher $1 \mathrm{~h}$ after RFA compared with those before RFA, and the difference was not significant. (C) TNF- $\alpha$ concentrations were significantly increased $1 \mathrm{~h}$ after RFA, and were 10-fold higher compared with those before RFA. TNF- $\alpha$ concentrations decreased after RFA and remained higher compared with those before RFA, and the difference was not significant. The peak value of TNF- $\alpha$ occurred $1 \mathrm{~h}$ after RFA. In the non-SIRS group, there were no significant differences in TNF- $\alpha$ level after RFA. Note: * indicates $P<0.05,{ }^{\star \star}$ indicates $P<0.01,{ }^{\star \star \star}$ indicates $P<0.001$, NS indicates no significance

Figure 2 

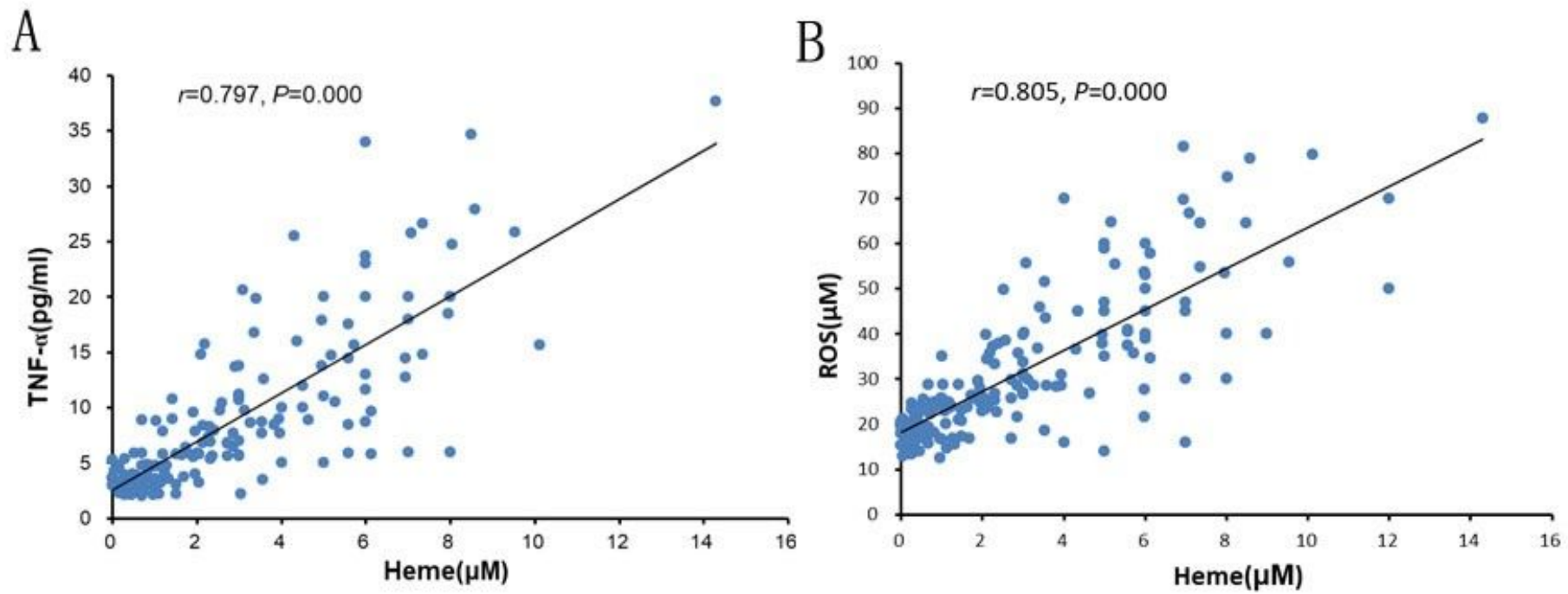

Figure 3. Pearson's correlation coefficients between heme and heme-related parameters. The concentrations circulating heme were significantly associated with those of ROS $(r=0.805, P<0.001)(A)$ and TNF-- $\alpha(r=0.797$, $P<0.001)(B)$.

Figure 3 